\title{
3n-Point Quaternary Shape Preserving Subdivision Schemes
}

\author{
MEHWISH BARI*, ROBINA BASHIR*, AND GHULAM MUSTAFA* \\ RECEIVED ON 07.03.2016 ACCEPTED ON 11.05.2016 \\ ABSTRACT
}

\begin{abstract}
In this paper, an algorithm is defined to construct $3 n$-point quaternary approximating subdivision schemes which are useful to design different geometric objects in the field of geometric modeling. We are going to establish a family of approximating schemes because approximating scheme provide maximum smoothness as compare to the interpolating schemes. It is to be observed that the proposed schemes satisfying the basic sum rules with bell-shaped mask go up to the convergent subdivision schemes which preserve monotonicity. We analyze the shape-preserving properties such that convexity and concavity of proposed schemes. We also show that quaternary schemes associated to the certain refinable functions with dilation $\mathbf{4}$ have higher order shape preserving properties. We also calculated the polynomial reproduction of proposed quaternary approximating subdivision schemes. The proposed schemes have tension parameter, so by choosing different values of the tension parameter we can get different limit curves of initial control polygon. We show in the table form that the proposed schemes are better than the existing schemes by comparing them on the behalf of their support and continuity. The visual quality of proposed schemes is demonstrated by different snapshots.
\end{abstract}

Key Words: Subdivision, Quaternary, Tension Control, Bell-Shaped Mask, Convexity, Concavity.

\section{INTRODUCTION}

$\mathrm{I}$ n computer graphics, subdivision technique constitutes a large class of recursive schemes for computing curves. The shape of control points that is the vertices of a polygonal curve plays a vital role in shape-preserving approximation which used in the design of curves to control their 'shape'.

First, approximating schemes were introduced by Rham [1] and Chaikin [2]. The well-known examples are the schemes which generate spline curves by Micchelli [3] which are bell-shaped of any arity (i.e. binary, ternary, quaternary,...n-ary). A lot of work has been done on binary subdivision schemes, but the research communities are taking interest to introduce higher arity schemes which give less computational cost and better result. There are numerous examples of binary and ternary monotonicity and convexity preserving subdivision schemes [4-10]. A similar approach to the cubic spline interpolation is convexity-preserving approximation method which is presented by Han [11]. He used the univariate cubic spline approximation function having $\mathrm{C}^{2}$ continuity. Mustafa and Bari [12] establish a family of odd point ternary nonstationary approximating schemes for conics. In [13], they also presented wide-ranging families of subdivision schemes for fitting data to subdivision models.

* Department of Mathematics, The Islamia University of Bahawalpur, Bahawalpur.

Mehran University Research Journal of Engineering \& Technology, Volume 36, No. 3, July, 2017 [p-ISSN: 0254-7821, e-ISSN: 2413-7219] 
Mustafa and Khan [14] proposed a 4-point quaternary approximating subdivision scheme with one shape parameter and analyzed its properties. Quaternary approximating schemes have higher smoothness and smaller support, comparing to binary and ternary schemes. So, the proposed quaternary subdivision schemes are improving the smoothness of the limit function while keeping a smaller support.

We are going to analyze the schemes having odd/even number of control points which led to a more general analyzable quaternary subdivision schemes. The most important result of the article is that the proposed family of schemes has monotonicity preserve as well as convexity and concavity preserve. Comparison of the smoothness of proposed schemes with existing schemes is also presented.

This paper is arranged as follows: Section 2 contains preliminaries. Shape preserving subdivision schemes of higher order is presented in Section 3. Polynomial reproduction, convexity and concavity preservation are also presented in Section 3. Numerical examples and comparison are presented in Section 4. Conclusion is presented in Section 5.

\section{PRELIMINARIES}

A quaternary subdivision scheme $S_{a}$ is defined by the subdivision rule:

$f_{i}^{k+1}=\sum_{j \in Z} a_{i-4 j} f_{j}^{k}, i \in Z$

Where $a=\left\{a_{\mathrm{i}} \in \mathrm{R}: \mathrm{i} \in \mathrm{Z}\right\}$ determines the mask of the scheme, $S_{a}$ is called subdivision scheme corresponding to the set of coefficients $a$. Throughout the work, we consider quaternary approximating scheme with a mask of finite support. We explicit a general formula for the mask of 3npoint symmetric subdivision scheme with one parameter that reproduce of degree $m=1$ (for linear reproduction). The quaternary subdivision scheme $S$ with the mask $\left\{a_{\mathrm{i}} \in \mathrm{R}: \mathrm{i} \in \mathrm{Z}\right\}$ necessarily satisfies

$\sum_{j \in Z} a_{4 j+q}=1, q=0,1,2,3$

Laurent polynomial is used as a symbol of subdivision scheme

$a(z)=\sum_{j \in Z} a_{j} z^{j}$

which can be written as $a(\mathrm{z})=\left(1+\mathrm{z}+\mathrm{z}^{2}+\mathrm{z}^{3}\right) b(\mathrm{z})$.

This implies

$b(z)=\sum_{j \in Z} b_{j} z^{j}=\left(1+z+z^{2}+z^{3}\right)^{-1} a(z)$

is a Laurent polynomial such that $b(1)=1$. By Albrecht and Romani [8], we write Equation (3) as:

$S_{b} \Delta \lambda=\Delta\left(S_{a} \lambda\right)$

where $S_{b}$ is subdivision operator associated with the difference mask $b=\left\{b_{j}\right\}_{j \in Z}$

$\Delta \lambda=\left\{\Delta \lambda_{j}\right\}_{j \in Z} \Delta \lambda_{j}=\lambda_{j}-\lambda_{j-1}$

If the subdivision scheme $S_{b}$ is contractive then the subdivision scheme $S_{a}$ is convergent. For $p \geq 0$ with $b(1)=1$ and

$b(z)=\frac{\left(1+z+z^{2}+z^{3}\right)^{p+1}}{4^{p}} a(z)$

where $b(\mathrm{z})$ is contractive, then $\psi_{a} \in \mathrm{C}^{p}(\mathrm{R})$ and

$\psi_{a}^{(r)}=S_{b^{r}}^{\infty} \delta, 1 \leq r \leq p$

where $S_{b^{r}}^{\infty}$ associated with the r-order

$b^{r}(\mathrm{z})=\left(1+\mathrm{z}+\mathrm{z}^{2}+\mathrm{z}^{3}\right)^{-r} 4^{r} a(\mathrm{z}), \quad 1 \leq r \leq p$. 
Definition 1. A sequence $\left\{a_{j}\right\}_{j=0}^{n=2 N+2}$ where $a_{j}=0$ for $j<0$ and $j>n$ is bell-shaped, if $a_{j}>0$ for $0 \leq j \leq n, a_{j}=a_{n-j}$ for 0 $\leq j \leq n, a_{0}<a_{1}<a_{2}<\ldots a_{[n / 2]}$ where [.] is bracket function.

Definition-2: By Mehaute and Uteras [15], given a set of control points $p_{i}^{k} \in Z, p_{i}^{k}=\left(x_{i}^{k}, f_{i}^{k}\right), f_{i}^{k}$ is strictly convex at a point $x_{i}^{k}$, if second order divided difference $d_{i}^{k}=f\left[p_{i-1}^{k}, x_{i}^{k}, x_{i-1}^{k}\right]>0$.

Definition-3: By Mehaute and Uteras [15], given a set of control points $p_{i}^{k} \in Z, p_{i}^{k}=\left(x_{i}^{k}, f_{i}^{k}\right), f_{i}^{k}$ is strictly concave at a point $x_{i}^{k}$, if second order divided difference $D_{i}^{k}=f\left[x_{i-1}^{k}, x_{i}^{k}, x_{i-1}^{k}\right]<0$.

The following theorem is followed by Pitolli [9].

Theorem-1: Any subdivision scheme related with bellshaped mask fulfilling the basic sum rules is monotonicity preserving.

Proof: Since $\boldsymbol{a}$ is compactly supported and satisfies Equation (1), Laurent polynomial $b(\mathrm{z})$ in Equation (2) has degree $3 N-1$. Thus, the mask sequence $b$ is compactly supported with suppb $=[0,3 N-1]$. The expression $a(\mathrm{z})=\left(1+\mathrm{z}+\mathrm{z}^{2}+\mathrm{z}^{3}\right) b(\mathrm{z})$.

$a_{j}=b_{j}+b_{j-1}+b_{j-2}, \quad 0 \leq j \leq 3 N+2$

From which, by induction, we get

$b_{4 j+q}=\sum_{i=0}^{j} \Delta a_{4 i+q}, q=0,1,2,3$

where $(\Delta a)_{j}=a_{j}-a_{j-1}>0$ for $0 \leq j \leq 3 n+2, n=1,3,5 \ldots$, for 3,6,9 point quaternary schemes respectively, so that $b_{j}>0$ for $0 \leq j \leq 3 n+1, n=1,3,5 \ldots$.

According the symmetric property of the mask, the equality in Equation (4) gives

$a_{j}=a_{(3 N+2)-j}=b_{3 N-j+2}+b_{3 N-j+1}+b_{3 N-j}+b_{3 N-j-1}, \quad 0 \leq j \leq 3 N+2$, from Equation (6) we get Equation (7)

$b_{3 N-j-1}=a_{j}-b_{3 N-j+2}-b_{3 N-j+1}-b_{3 N-j}, \quad 0 \leq j \leq 3 N-1$

Recursively, we find that for $j=0,1,2, \ldots, N, b_{3 N-j-1}=b_{j}$ and the claim follows.

When $a(\mathrm{z})$ is bell-shaped and has a factor $\left(1+\mathrm{z}+\mathrm{z}^{2}+\right.$ $\left.z^{3}\right)^{2}$, then bell-shaped $b^{1}(z)$ satisfies the basic sum rules. Thus, Laurent polynomial

$c(\mathrm{z})=\left(1+\mathrm{z}+\mathrm{z}^{2}+\mathrm{z}^{3}\right)^{-1} b^{1}(\mathrm{z})$.

Equation (8) has the degree $3 N-4$, and

$b_{j}^{1}=c_{j}+c_{j-1}+c_{j-2}+c_{j-3}, 0 \leq j \leq 3 N-1$

From Equation (9) we get Equation (10)

$c_{4 j+q}=\sum_{i=0}^{j} \Delta b_{4 i+q}^{1}, q=0,1,2,3$

Since $b^{1}$ holds bell-shape property so the mask $c$ is symmetric and positive.

\section{HIGHER ORDER SHAPE PRESERVING SUBDIVISION SCHEMES}

Subdivision schemes preserving shape with higher order can be expressed as:

$\boldsymbol{a}_{\beta, N}=\left\{a_{i, \beta, N}\right\}_{1 \leq i \leq 3(N+1),}$

where

$a_{i, \beta, N}=\frac{\beta}{4}\left\{b_{i, N-1}+\left(\frac{4}{\beta}-2\right) b_{i-1, N-1}+b_{i-2, N-1}\right\}$

$0 \leq \beta \leq 2$ is a shape parameter and $N=3,7,11,15, \ldots$ in Equation (11). The subdivision scheme $S_{a, \beta, N}$ converges and generate $C^{N-1}$ limit function for $0 \leq \beta \leq 2$. The divided difference masks of the symbol

$$
\begin{aligned}
& a_{\beta, N}(z)=\frac{1}{4^{N}}\left(1+z+z^{2}+z^{3}\right)^{N}\left(\beta z^{2}+(4-2 \beta) z+\beta\right) \\
& b_{\beta, N}^{r}(z)=\left(1+z+z^{2}+z^{3}\right)^{-r} 4^{r} a_{\beta, N}(z), 1 \leq r \leq N
\end{aligned}
$$


bell-shaped. So, the subdivision scheme $S_{a, \beta, N}$ has shapepreserving properties of order $N$, i.e. $S_{a, \beta, N}$ conserves the sign of the first-order difference $\Delta^{r}$ f for $1 \leq r \leq N$, where $\Delta^{r} \mathrm{f}=\Delta\left(\Delta^{r-1} \mathrm{f}\right)$. The subdivision scheme $S_{a, \beta, N}$ has best shape-preserving properties in the logic that it conserves the utmost with respect to the mask support with achievable order differences. From Equation (12), the masks of 3-point $(N=3)$ and 6-point $(N=7)$ quaternary schemes will be

$a_{\beta, 3}(\mathrm{z})=1 / 4^{3}\left\{\beta+(4+\beta) \mathrm{z}+(12+\beta) \mathrm{z}^{2}+(24+\beta) \mathrm{z}^{3}+(40-2 \beta) \mathrm{z}^{4}+(48-2 \beta) \mathrm{z}^{5}\right.$
$\left.+(48-2 \beta) \mathrm{z}^{6}+(40-2 \beta) \mathrm{z}^{7}+(24+\beta) \mathrm{z}^{8}+(12+\beta) \mathrm{z}^{9}+(4+\beta) \mathrm{z}^{10}+\beta \mathrm{z}^{11}\right\}$

and

$a_{\beta, 7}(\mathrm{z})=1 / 4^{7}\left\{\beta+(4+5 \beta) \mathrm{z}+(28+15 \beta) \mathrm{z}^{2}+(112+35 \beta) \mathrm{z}^{3}+\right.$ $(336+63 \beta) \mathrm{z}^{4}+(812+91 \beta) \mathrm{z}^{5}+(1652+105 \beta) \mathrm{z}^{6}+$ $(2912+85 \beta) z^{7}+(4512+26 \beta) z^{8}+(6216-62 \beta) z^{9}+(7672-$ $154 \beta) z^{10}+(8512-210 \beta) z^{11}+(8512-210 \beta) z^{12}+(7672-$ $154 \beta) \mathrm{z}^{13}+(6216-62 \beta) \mathrm{z}^{14}+(4512+26 \beta) \mathrm{z}^{15}+(2912+85 \beta) \mathrm{z}^{16}$ $+(1652+105 \beta) \mathrm{z}^{17}+(812+91 \beta) \mathrm{z}^{18}+(336+63 \beta) \mathrm{z}^{19}+$ $\left.(112+35 \beta) \mathrm{z}^{20}+(28+15 \beta) \mathrm{z}^{21}+(4+5 \beta) \mathrm{z}^{22}+\beta \mathrm{z}^{23}\right\}$.

The mask of 3-point quaternary approximating scheme in [16] coincide with $a_{\beta, 3}$ when $\beta=1 / 2$ and this scheme gives $C^{2}$ continuity. The mask $a_{\beta, 7}$ gives $C^{6}$ continuity. Similarly $a_{\beta, 11}$ gives $C^{10}$ continuity. We will derive explicit formulae for the mask of $3 n$-point quaternary subdivision schemes which are bell-shaped compactly supported.

Definition 3.1: By Siddiqi and Younis [16], for quaternary subdivision scheme the parameterization $\tau_{a}=\frac{a^{\prime}(1)}{4}$, the corresponding parametric shift and attach the data $f_{i}^{k}$ for $i \in \mathrm{Z}, k \in N$ to the parameter values

$t_{i}^{k}=t_{0}^{k}+\frac{i}{4^{k}}$ with $t_{0}^{k}=t_{0}^{k-1}-\frac{\tau}{4^{k}}$

Theorem-2: A convergent quaternary subdivision scheme reproduces polynomials of degree $\mathrm{m}$ with respect to the parameterization defined in Equation (13) if.

$$
a^{(k)}(1)=4 \bigsqcup_{l=0}^{k-1}(\tau-1) \text { and } a^{(k)}\left(e^{\frac{2 n i \Pi}{4}}\right)=0
$$

for $n=1,2,3$ and $k=0,1, \ldots \mathrm{m}$.

Proof: The induction over $\mathrm{m}$ can be performed to prove this theorem following [17].

The necessary conditions defined in Equation (14) will be found with the help of following proposition, by view of [17].

Proposition-1. If $\mathrm{m} \in N$ and $\tau \in \mathrm{R}$, then a subdivision symbol $a(\mathrm{z})$ satisfies

$a^{(k)}(1)=4 \bigsqcup_{l=0}^{k-1}(\tau-l)$ for $\mathrm{k}=1,2, \ldots \mathrm{m}$

If.. $b(\mathrm{z})=a\left(\mathrm{z}^{4}\right) \mathrm{z}^{-4 \tau}$ satisfies $b(1)=4$ and $b^{(k)}(1)=0$ for $k=$ $0,1, \ldots, \mathrm{m}$.

Proposition-2: If a quaternary subdivision scheme that reproduces polynomial up to degree $m$ then the smoothed scheme $S_{b}$ with the symbol

$b(z)=\frac{1+z+z^{2}+z^{3}}{4} a(z)$

assures the conditions $b(1)=4$ and $b^{(k)}\left(\mathrm{e}^{2 n i \Pi / 4}\right)=0$ for $k=0,1, \ldots, \mathrm{m}+1$ and hence generates polynomial of degree $\mathrm{m}+1$ but it has only linear reproduction .

Proof: For some symbol $b(\mathrm{z})$ with $b(1)=1 / 4^{\mathrm{m}}$, we have $a(\mathrm{z})=\left(1+\mathrm{z}+\mathrm{z}^{2}+\mathrm{z}^{3}\right)^{\mathrm{m}+1} b(\mathrm{z})$

Taking first derivative of $b(\mathrm{z})$ is

$b^{\prime}(z)=\frac{1+z+z^{2}+z^{3}}{4} a^{\prime}(z)+\frac{1+2 z+3 z^{3}}{4} a(z)$

since

$\tau_{b}=\frac{b^{\prime}(1)}{4}=\frac{1}{4} a^{\prime}(1)+\frac{3}{8} a(1)=\tau_{a}+\frac{3}{2}$ 
The second derivative is

$b^{\prime \prime}(z)=\frac{1+z+z^{2}+z^{3}}{4} a^{\prime \prime}(z)+\frac{1+2 z+3 z^{2}}{4} a^{\prime}(z)+\frac{2+6 z}{4} a(z)$

and

$b^{\prime \prime}(1)=a^{\prime \prime}(1)+3 a^{\prime}(1)+2 a(1)=4 \tau_{a}\left(\tau_{a}-1\right)+12 \tau_{a}+8$

simplifying, we get

$b^{\prime \prime}(1)=4 \tau_{b}\left(\tau_{b}-1\right) \neq 0$

So, the proposed scheme reproduces polynomial of degree $m=1$.

\section{CONVEXITY PRESERVATION}

We prove the convexity preservation of subdivision schemes $S_{a, \beta, 3}$ with uniform initial control points. Given a set of initial control points $p_{i}^{0} \in Z, p_{i}^{0}=\left(x_{i}^{0}, f_{i}^{0}\right)$ which are strictly convex, where $x_{i}^{0} \in Z$ are equidistant points. To simplify, we use $\Delta x_{i}^{0}=x_{i+1}^{0}-x_{i}^{0}=1$. By the subdivision scheme $S_{a, \beta, 3}$, we have

$\Delta x_{i}^{k+1}=x_{i+1}^{k+1}-x_{i}^{k+1}=\frac{1}{4} \Delta x_{i}^{k}=\frac{1}{4^{k+1}}$

Denote $d_{i}^{k}=f\left[x_{i-1}^{k}, x_{i}^{k}, x_{i+1}^{k}\right]=4^{2 k} 2^{-1}\left(f_{i-1}^{k}-2 f_{i}^{k}+f_{i+1}^{k}\right)$ as the second order divided differences. In the following, we will prove $d_{i}^{k}>0 \forall k \geq 0, k \in Z, i \in Z$

Theorem-3: Suppose that the initial control points $\left\{p_{i}^{0}\right\}_{i \in Z},\left\{p_{i}^{0}\right\}=\left(x_{i}^{0}, f_{i}^{0}\right)$ are strictly convex, i.e. $d_{i}^{k}>0$, for all $i \in Z$

$d_{i}^{k}=4^{2 k}(2)^{-1}\left(f_{i-1}^{k}-2 f_{i}^{k}+f_{i-1}^{k}\right), y_{i}^{k}=\frac{d_{i+1}^{k}}{d_{i}^{k}}$

$Y^{k}=\max _{i}\left\{y_{i}^{k}, \frac{1}{y_{i}^{k}}\right\}, \forall k \geq 0, k \in Z, i \in Z$
Furthermore, let $0.1 \leq \beta \leq 0.9$ and $\delta=5+5 \beta$, $\delta \in \mathrm{R}$. Then for $1 / \delta \leq Y^{0} \leq \delta$,

$d_{i}^{k}>0, \frac{1}{\delta} \leq Y^{k} \leq \delta, \forall k \geq 0, k \in Z, i \in Z$

That is, the limit function generated by the subdivision scheme $S_{a, \beta, 3}$ is strictly convex.

Proof. To prove Equation (15), we will use mathematical induction. When $k=0, d_{i}^{0}>0, \frac{1}{\delta} \leq Y^{0} \leq \delta$, then Equation (15) is true. Suppose that the Equation (15) holds for $k$. i.e. $d_{i}^{k}>0, \frac{1}{\delta} \leq Y^{k} \leq \delta$, next we will prove that the Equation (15) hold for $k+1$. Since

$d_{4 i}^{k+1}=4^{2 k}(2)^{-1}\left(f_{4 i-1}^{k+1}-2 f_{4 i}^{k+1}+f_{4 i+1}^{k+1}\right)$

This implies that

$d_{4 i}^{k+1}=\frac{\beta}{4} d_{4 i-1}^{k}+\left(1-\frac{\beta}{4}\right) d_{i}^{k}$

Similarly

$d_{4 i+1}^{k+1}=d_{i}^{k}$

$d_{4 i+2}^{k+1}=d_{i}^{k}$

$d_{4 i+3}^{k+1}=\left(1-\frac{\beta}{4}\right) d_{i-1}^{k}+\frac{\beta}{4} d_{i}^{k}$

Next we show that

$d_{4 i}^{k+1}>0, d_{4 i+1}^{k+1}>0, d_{4 i+2}^{k+1}>0$ and $d_{4 i+3}^{k+1}>0$

Since

$d_{4 i}^{k+1}=d_{i}^{k}\left\{\frac{\beta}{4} \frac{1}{y_{i-1}^{k}}+\left(1-\frac{\beta}{4}\right)\right\}$

Then

$d_{4 i}^{k+1}=d_{i}^{k}\left\{\frac{\beta}{4} \frac{1}{\delta}+\left(1-\frac{\beta}{4}\right)\right\}$ 
As we know that $d_{i}^{k}>0$ and

$\left\{\frac{\beta}{4} \frac{1}{\delta}+\left(1-\frac{\beta}{4}\right)\right\}>0$

for $0.1 \leq \beta \leq 0.9$ and $\delta=5+6 \beta$.

This implies that

$d_{4 i}^{k+1}>0$

Similarly, we can prove that

$d_{4 i+1}^{k+1}>0, d_{4 i+2}^{k+1}>0$

and

$d_{4 i+3}^{k+1}>0$

for $0.1 \leq \beta \leq 0.9$ and $\delta=5+6 \beta$.

Now we prove that

$\frac{1}{\delta} \leq Y^{k+1} \leq \delta$

we first show that

$y_{4 i}^{k+1}-\delta \leq 0$

Since

$y_{4 i}^{k+1}-\delta=\frac{d_{4 i+1}^{k+1}}{d_{4 i}^{k+1}}-\delta=\frac{d_{i}^{k}-\frac{\beta}{4} d_{i-1}^{k} \delta-\left(1-\frac{\beta}{4}\right) d_{i}^{k} \delta}{\frac{\beta}{4} d_{i-1}^{k}+\left(1-\frac{\beta}{4}\right) d_{i}^{k}}$

This implies that

$y_{4 i}^{k+1}-\delta \leq \frac{\left\{1-\frac{\beta}{4}-\left(1-\frac{\beta}{4}\right) \delta\right\}}{\frac{\beta}{4} \delta+\left(1-\frac{\beta}{4}\right)}$
The denominator and numerator of right hand side of above expression are greater than and less than zero respectively for $0.1 \leq \beta \leq 0.9$ and $\delta=5+6 \beta$. This implies that

$y_{4 i}^{k+1}-\delta \leq 0$

Further implies that

$y_{4 i}^{k+1} \leq \delta$

Now we show that

$\frac{1}{y_{4 i}^{k+1}}-\delta \leq 0$

Since

$\frac{1}{y_{4 i}^{k+1}}-\delta=\frac{d_{4 i}^{k+1}}{d_{4 i+1}^{k+1}}-\delta=\frac{\frac{\beta}{4} d_{i-1}^{k}+\left(1-\frac{\beta}{4}\right) d_{i}^{k}-d_{i}^{k} \delta}{d_{i}^{k}}$

This implies that

$\frac{1}{y_{4 i}^{k+1}}-\delta \leq\left\{\left(\frac{\beta}{4}-1\right) \delta+1-\frac{\beta}{4}\right\}$

Since

$\left\{\left(\frac{\beta}{4}-1\right) \delta+1-\frac{\beta}{4}\right\} \leq 0$

for $0.1 \leq \beta \leq 0.9$ and $\delta=5+6 \beta$.

This implies that

$\frac{1}{y_{4 i}^{k+1}}-\delta \leq 0$

Further implies that

$\frac{1}{y_{4 i}^{k+1}} \leq \delta$ 
In the same way, we see that

$$
y_{4 i+1}^{k+1} \leq \delta, y_{4 i+2}^{k+1} \leq \delta, y_{4 i+3}^{k+1} \leq \delta, \frac{1}{y_{4 i+1}^{k+1}} \leq \delta, \frac{1}{y_{4 i+2}^{k+1}} \leq \delta \text { and } \frac{1}{y_{4 i+3}^{k+1}} \leq \delta
$$

Since

$$
Y^{k+1}=\max _{i}\left\{y_{i}^{k+1}, \frac{1}{y_{i}^{k+1}}\right\}
$$

it is obvious that

$$
Y^{k+1} \geq \frac{1}{\delta}
$$

This completes the proof.

\subsection{Concavity Preservation}

We prove the concavity preservation of subdivision schemes $S_{a, \beta, 3}$ with uniform initial control points.

Theorem-4: Suppose that the initial control points $\left\{p_{i}^{0}\right\}_{i \in Z},\left\{p_{i}^{0}\right\}=\left(x_{i}^{0}, f_{i}^{0}\right)$ are strictly concave, i.e.

$D_{i}^{0}<0$, for all $\mathrm{i} \in \mathrm{Z}$. Let

$D_{i}^{k}=4^{2 k}(2)^{-1}\left(f_{i+1}^{k}-2 f_{1}^{k}+f_{i+1}^{k}\right), q_{1}^{k}=\frac{D_{i+1}^{k}}{D_{i}^{k}}$

$Q^{k}=\max _{i}\left[q_{i}^{k}, \frac{1}{q_{i}^{k}}\right], \forall k \geq 0, k \in Z, i \in Z$

Furthermore, let $0.1 \leq \beta \leq 0.9$ and $\lambda=5+5 \beta$, $\lambda \in \mathrm{R}$. Then for $1 / \lambda \leq Q^{0} \leq \lambda$,

$D_{i}^{k}<0, \frac{1}{\lambda} \leq Q^{k} \leq \lambda, \forall k \geq 0, k \in Z, i \in Z$

That is, the limit function generated by the subdivision scheme $\mathrm{S}_{\mathrm{a}, \beta, 3}$ is strictly concave.

Proof. To prove Equation (16), we will use mathematical induction. If

$$
k=0, D_{i}^{k}<0, \frac{1}{\lambda} \leq Q^{o} \leq \lambda
$$

then the Equation (16) is true. Suppose that the Equation (16) holds for $k$. i.e.

$$
D_{i}^{k}<0, \frac{1}{\lambda} \leq Q^{k} \leq \lambda
$$

Next we will prove that the Equation (16) hold for $k+1$. Since

$$
D_{4 i}^{k+1}=4^{2 k}(2)^{-1}\left(f_{4 i-1}^{k+}-2 f_{4 i}^{k+1}+f_{4 i+1}^{k+1}\right)
$$

This implies that

$$
D_{4 i}^{k+1}=\frac{\beta}{4} D_{i-1}^{k+1}+\left(1-\frac{\beta}{4}\right) D_{i}^{k}
$$

Similarly

$$
\begin{aligned}
& D_{4 i+1}^{k+1}=D_{i}^{k} \\
& D_{4 i+2}^{k+1}=D_{i}^{k} \\
& D_{4 i+3}^{k+1}=\left(1-\frac{\beta}{4}\right) D_{i-1}^{k}+\frac{\beta}{4} D_{i}^{k}
\end{aligned}
$$

Next we show that

$$
D_{4 i}^{k+1}<0, D_{4 i+1}^{k+1}<0, D_{4 i+2}^{k+1}<0 \text { and } D_{4 i+3}^{k+1}<0
$$

Since

$$
D_{4 i}^{k+1}=D_{i}^{k}\left\{\frac{\beta}{4} \frac{1}{q_{i-1}^{k}}+\left(1-\frac{\beta}{4}\right)\right\}
$$

Then

$$
D_{4 i}^{k+1} \leq D_{i}^{k}\left\{\frac{\beta}{4} \lambda+\left(1-\frac{\beta}{4}\right)\right\}
$$

As we know that $D_{i}^{k}<0$ and

$$
\left\{\frac{\beta}{4} \lambda+\left(1-\frac{\beta}{4}\right)\right\}>0
$$


for $0.1 \leq \beta \leq 0.9$ and $\lambda=5+6 \beta$.

This implies that

$D_{4 i}^{k+1}<0$

Similarly, we can prove that

$D_{4 i+1}^{k+1}<0, D_{4 i+2}^{k+1}<0$ and $D_{4 i+3}^{k+1}<0$ for $0.1 \leq \beta \leq 0.9$ and $\lambda=5+6 \beta$

Now we prove that

$\frac{1}{\lambda} \leq Q^{k+1} \leq \lambda$

We first show that

$q_{4 i}^{k+1}-\lambda \leq 0$

Since

$q_{4 i}^{k+1}-\lambda=\frac{D_{4 i+1}^{k+1}}{D_{4 i}^{k+1}}-\lambda=\frac{D_{i}^{k}-\frac{\beta}{4} D_{i-1}^{k} \lambda-\left(1-\frac{\beta}{4}\right) D_{i}^{k} \lambda}{\frac{\beta}{4} D_{i-1}^{k}+\left(1-\frac{\beta}{4}\right) D_{i}^{k}}$

This implies that

$q_{4 i}^{k+1}-\lambda \leq \frac{\left\{1-\frac{\beta}{4}-\left(1-\frac{\beta}{4}\right) \lambda\right\}}{\frac{\beta}{4} \lambda+\left(1-\frac{\beta}{4}\right)}$

The denominator and numerator of right hand side of above expression are greater than and less than zero respectively for $0.1 \leq \beta \leq 0.9$ and $\lambda=5+6 \beta$. This implies that

$q_{4 i}^{k+1}-\lambda \leq 0$

Further implies that

$q_{4 i}^{k+1} \leq \lambda$

Now we show that

$$
\frac{1}{q_{4 i}^{k+1}}-\lambda \leq 0
$$

$\frac{1}{q_{4 i}^{k+1}}-\lambda=\frac{D_{4 i}^{k+1}}{D_{4 i+1}^{k+1}}-\lambda=\frac{\frac{\beta}{4} D_{i-1}^{k}+\left(1-\frac{\beta}{4}\right) D_{i}^{k}-D_{i}^{k} \lambda}{D_{i}^{k}}$

This implies that

$\frac{1}{q_{4 i}^{k+1}}-\lambda \leq\left\{\left(\frac{\beta}{4}-1\right) \lambda+1-\frac{\beta}{4}\right\}$

Since

$\left\{\left(\frac{\beta}{4}-1\right) \lambda+1-\frac{\beta}{4}\right\}<0$

for $0.1 \leq \beta \leq 0.9$ and $\lambda=5+6 \beta$.

This implies that

$\frac{1}{q_{4 i}^{k+1}}-\lambda \leq 0$

Further implies that

$\frac{1}{q_{4 i}^{k+1}} \leq \lambda$

In the same way, we see that

$\frac{1}{q_{4 i+1}^{k+1}} \leq \lambda, \frac{1}{q_{4 i+2}^{k+1}} \leq \lambda, \frac{1}{q_{4 i+3}^{k+1}} \leq \lambda$

So

$Q^{k+1} \leq \lambda$

Since

$Q^{k+1}=\max _{i}\left\{q_{i}^{k+1}, \frac{1}{q_{i}^{k+1}}\right\}$

it is obvious that

$Q^{k+1} \geq \frac{1}{\lambda}$

This completes the proof. 


\section{NUMERICAL EXAMPLES AND COMPARISON}

Figs. 1-2 are produced by using convex and concave data set given in Tables 1-2 respectively. We choose the

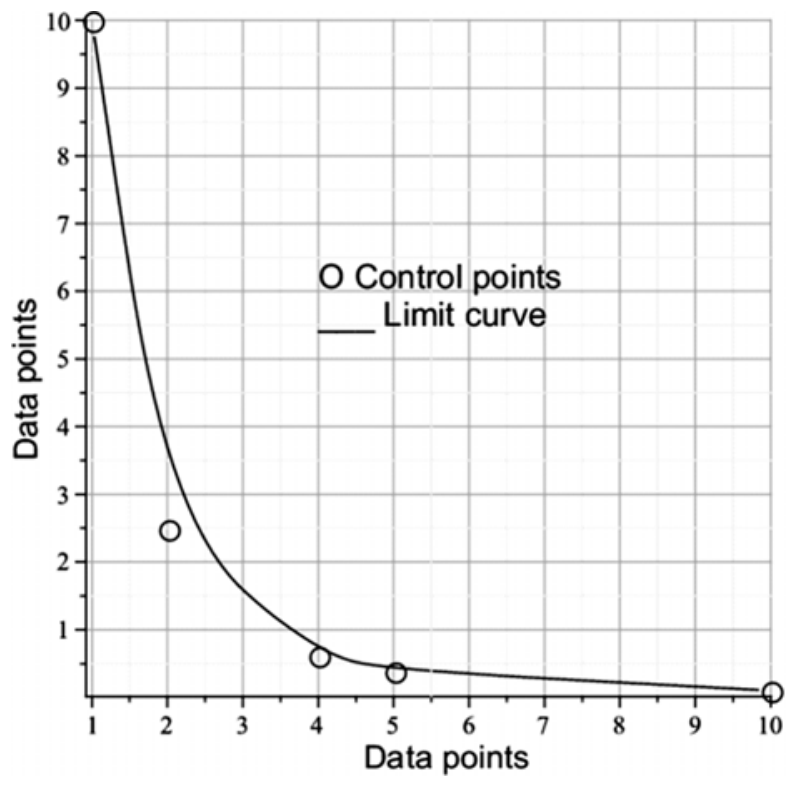

(a) Convexity of 3-Point Quaternary Scheme

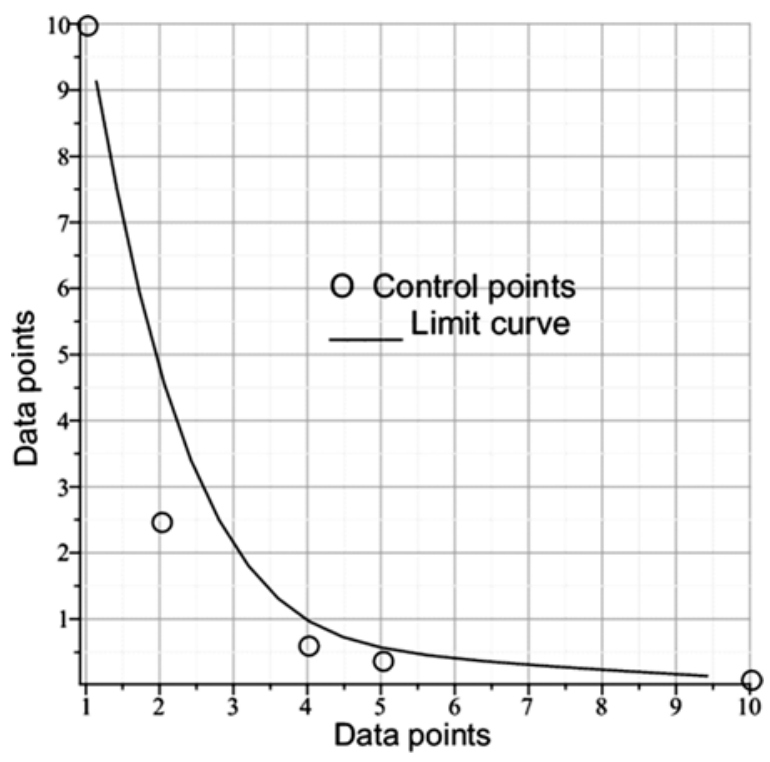

(b) Convexity of 6-Point Quaternary Scheme

FIG. 1. THE CONVEX CURVES GENERATED BY SCHEMES $S_{a, \beta, 3}$ AND $S_{a, \beta, 7} R E S P E C T I V E L Y$ convex data set by Sarfraz [18]. Convex and concave curves are produced by the schemes $\mathrm{S}_{\mathrm{a}, \beta, 3}$ and $\mathrm{S}_{\mathrm{a}, \beta, 7}$ are shown in Fig. 1(a-b) and Fig. 2(a-b) respectively at $\beta=0.5$. Fig. 3(a-b) shows the behavior of proposed schemes

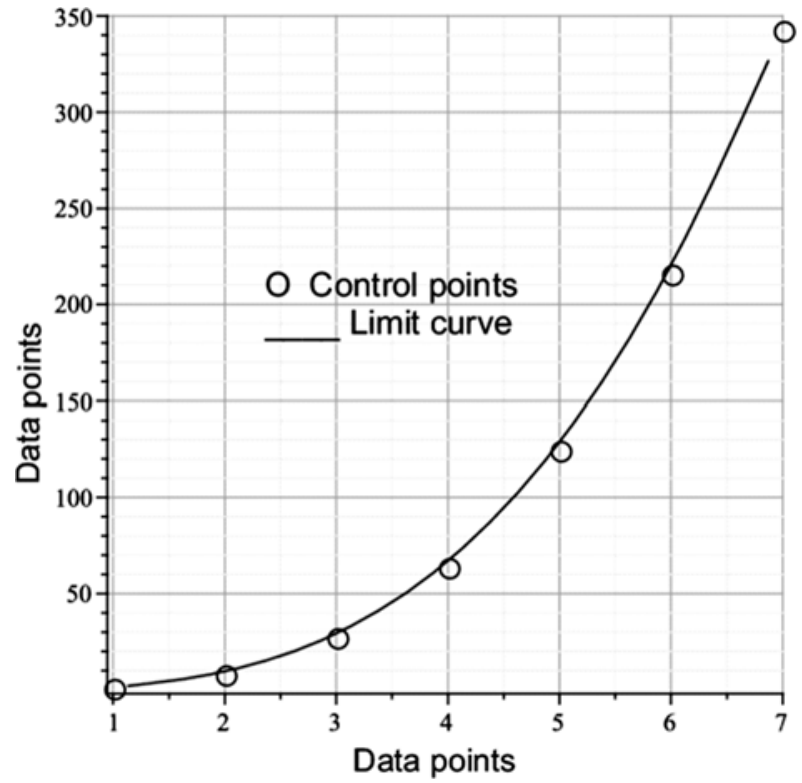

(a) Conveavity of 3-Point Quaternary Scheme

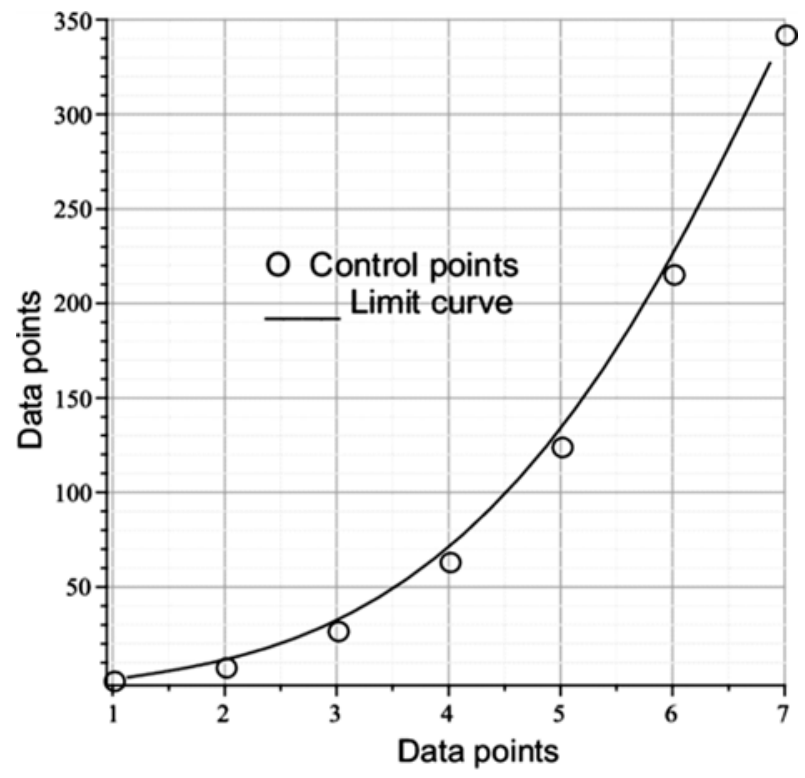

(b) Conveavity of 6-Point Quaternary Scheme

FIG. 2. THE CONCAVE CURVES GENERATED BY SCHEMES $S_{a, \beta, 3}$ AND $S_{a, \beta, 7} R E S P E C T I V E L Y$ 
$S_{a, \beta, 3}$ and $S_{a, \beta, 7}$ at the different values of tension parameter $\beta$. Table 3 shows the comparison of shape preserving quaternary approximating schemes with existing schemes.

TABLE 1. CONVEX DATA SET

\begin{tabular}{|c|c|c|c|c|c|}
\hline (x,y) & \multicolumn{5}{|l|}{} \\
\hline $\mathrm{x}$ & 1 & 2 & 4 & 5 & 10 \\
\hline $\mathrm{y}$ & 10 & 2.5 & 0.065 & 0.4 & 0.1 \\
\hline
\end{tabular}

TABLE 2. CONCAVE DATA SET

\begin{tabular}{|c|c|c|c|c|c|}
\hline (x,y) & \multicolumn{5}{|l|}{} \\
\hline $\mathrm{x}$ & 1 & 2 & 3 & 4 & 5 \\
\hline $\mathrm{y}$ & 1 & 8 & 27 & 64 & 125 \\
\hline
\end{tabular}

TABLE 3. CONTINUITY AND SUPPORT OF PROPOSED SCHEMES WITH EXISTING SCHEMES

\begin{tabular}{|c|c|c|}
\hline Schemes & Support & Continuity \\
\hline 4-Point BinaryApproximating [19] & 7 & 2 \\
\hline 3-Point Ternary Approximating [20] & 4 & 2 \\
\hline 4-Point Ternary Approximating [21] & 5.5 & 2 \\
\hline 4-Point Quaternary [22] & 5 & 2 \\
\hline 4-Point Quaternary [23] & 5 & 3 \\
\hline 3-Point Quaternary Proposed & 3.6 & 2 \\
\hline 6-Point Quaternary Proposed & 7.6 & 6 \\
\hline 9-Point Quaternary Proposed & 13 & 10 \\
\hline
\end{tabular}

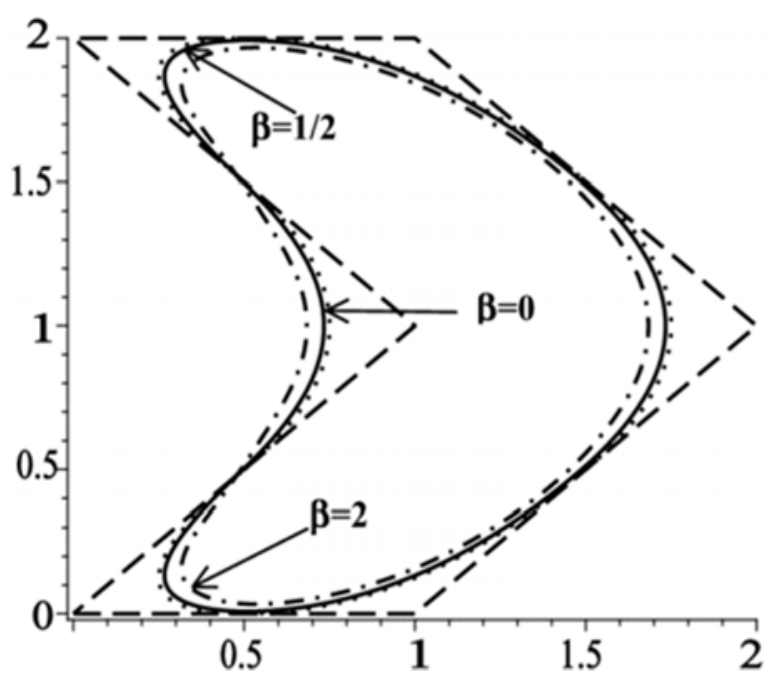

(a) Effect of Parameter of Proposed 3-Point Quaterney Approximating Scheme

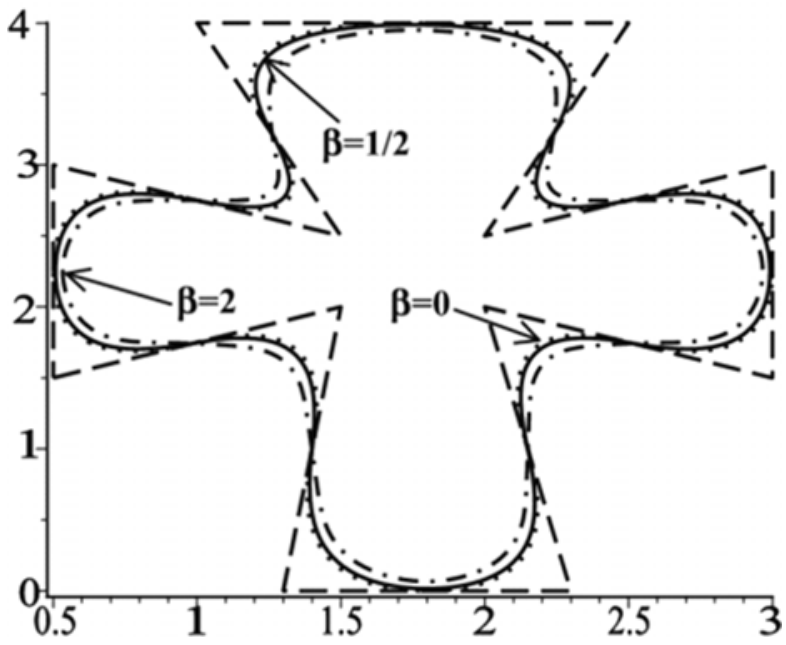

(b) Effect of Parameter of Proposed 6-Point Quaterney Approximating Scheme

FIG. 3. THE TIGHTNESS AND LOOSENESS OF THE CURVE BY CHANGING THE VALUE OF $\beta$

\section{CONCLUSION}

A family of 3n-point quaternary shape preserving subdivision scheme with a tension parameter has been discussed which generate smooth limiting curves. The main goal is to establish the quaternary schemes with higher smoothness and smaller support as comparing to lower arity schemes. The convexity, concavity, polynomial reproduction and visual smoothness of proposed schemes are also discussed. 


\section{ACKNOWLEDGEMENT}

This work is supported by The Islamia University of Bahawalpur, Pakistan, and NRPU Project No. 3183 from

Higher Education Commission (HEC) and the Indigenous Ph. D 5000 Fellowship Program..

\section{REFERENCES}

[1] Rham, de.G., "Un Peude Mathematiques a Proposed Une Courbe Plane”, Revwed Mathematiques Elementry-II, Oevred Completes, pp. 678-689, 1947.

[2] Chaikin, G.M., “An Algorithm for High-Speed Curve Generation”, Computer Graphics and Image Processing, Volume 3, No. 4, pp. 346-349, 1974.

Cai, Z., "Convexity Preservation of the Interpolating Four-Point $C^{2}$ Ternary Stationary Subdivision Scheme”, Computer Aided Geometric Design, Volume 26, pp. 560-565, 2009.

[8] Albrecht, G., and Romani, L., “Convexity Preserving Interpolatory Subdivision with Conic Precision”, Applied Mathematics and Computation, Volume 219, pp. 4049-4066, 2012.
[9] Pitolli, F., "Ternary Shape-Preserving Subdivision Schemes”, Mathematics and Computers in Simulation, Volume 106, pp. 185-194, 2013.

[10] Tan, J., Zhuang, X., and Zhang, L., "A New Four-Point Shape-Preserving $\mathrm{C}^{3}$ Subdivision Scheme”, Computer Aided Geometric Design, Volume 31, pp. 57-62, 2014.

[11] Han, X., "Convexity-Preserving Approximation by Univariate Cubic Splines”, Journal of Computational and Applied Mathematics, Volume 287, pp. 196-206, 2015.

[12] Mustafa, G., and Bari, M., "A New Class of Odd-Point Ternary Non-Stationary Approximating Schemes", Boletín de la Sociedad Española de Matemática Aplicada, Volume 68, No. 1, pp. 29-51, 2015.

[13] Mustafa, G., and Bari, M., "Wide-Ranging Families of Subdivision Schemes for Fitting Data”, Punjab University Journal of Mathematics, Volume 48, No. 2, pp. 125-134, 2016.

[14] Mustafa, G., and Khan, F., "A New 4-Point $C^{3}$ Quaternary Approximating Subdivision Scheme”, Abstract and Applied Analysis, pp. 14, 2009.

[15] Mehaute, A.L., and Uteras, F.I., "Convexity-Preserving Interpolatorty Subdivision”, Computer Aided Geometric Design, Volume 11, pp. 17-37, 1994.

[16] Siddiqi, S.S., and Younis, M., “The M-Point Quaternary Approximating Subdivision Schemes", American Journal of Computational Mathematics, Volume 3, pp. 6-10, 2013.

[17] Conti, C., and Hormann, K., "Polynomial Reproduction for Univariate Subdivision Schemes of Any Arity”, Journal of Approximation Theory, Volume 163, pp. 413437, 2011.

[18] Sarfraz, M., "Visualization of Positive and Convex Data by a Rational Cubic Spline Interpolation”, Information Sciences, Volume 146, pp. 239-254, 2002.

[19] Dyn, N., Floater, M.S., and Hormann, K., "A C ${ }^{2}$ FourPoint Subdivision Scheme with Fourth Order Accuracy and Its Extension”, Mathematical Methods for Curves and Surfaces, Daehlen, M., Morken, K., and Schumaker, L.L., (Editors), pp. 145-156, Tromso 2004. 
[20] Mustafa, G., Ghaffar, A., and Khan, F., "The Odd-Point Ternary Approximating Schemes”, American Journal of Computational Mathematics, Volume 1, No. 2, pp. 111-118, 2011.

[21] Ko, K.P., Lee, B.G., and Yoon, G.J., “A Ternary 4-Point Approximating Subdivision Scheme”, Applied Mathematics and Computation, Volume 190, pp. 1563-1573, 2007.
Ko, K.P., “A Quaternary Approximating 4-Point Subdivision Scheme”, The Journal of the Korean Society for Industrial and Applied Mathematics, Volume 13, No. 4, pp. 307-314, 2009.

[23] Mustafa, G., Khan, F., and Ghaffar, A., “The M-Point Approximating Subdivision Scheme”, Lobachevskii Journal of Mathematics, Volume 30, No. 2, pp. 138-145, 2009.

Mehran University Research Journal of Engineering \& Technology, Volume 36, No. 3, July, 2017 [p-ISSN: 0254-7821, e-ISSN: 2413-7219] 